\title{
Headaches precipitated by cough, prolonged exercise or sexual activity: a prospective etiological and clinical study
}

\author{
Julio Pascual · Andrés González-Mandly • \\ Rubén Martín · Agustín Oterino
}

Received: 30 May 2008/ Accepted: 17 July 2008/Published online: 28 August 2008

(C) Springer-Verlag 2008

\begin{abstract}
Headaches provoked by cough, prolonged physical exercise and sexual activity have not been studied prospectively, clinically and neuroradiologically. Our aim was to delimitate characteristics, etiology, response to treatment and neuroradiological diagnostic protocol of those patients who consult to a general Neurological Department because of provoked headache. Those patients who consulted due to provoked headaches between 1996 and 2006 were interviewed in depth and followed-up for at least 1 year. Neuroradiological protocol included craniocervical MRI for all patients with cough headache and dynamic cerebrospinal functional MRI in secondary cough headache cases. In patients with headache provoked by prolonged physical exercise or/and sexual activity cranial neuroimaging (CT and/or MRI) was performed and, in case of suspicion of subarachnoid bleeding, angioMRI and/or lumbar tap were carried out. A total of 6,412
\end{abstract}

Enrico Greppi Committee: Lorenzo Pinessi, Fabio Antonaci, Paolo Martelletti, Michael A. Moskowitz, Rigmor Jensen, Francesco Pierelli.

J. Pascual $(\square)$

Service of Neurology, University Hospital of Salamanca, 37007 Salamanca, Spain

e-mail: juliopascual@usal.es; juliopascual@telefonica.net

A. González-Mandly

Section of Neuroradiology, University Hospital Marqués de Valdecilla, Santander, Spain

R. Martín

Service of Neurosurgery, University Hospital Marqués de Valdecilla, Santander, Spain

A. Oterino

Service of Neurology, University Hospital Marqués

de Valdecilla, Santander, Spain patients consulted due to headache during the 10 years of the study. The number of patients who had consulted due to any of these headaches is 97 (1.5\% of all headaches). Diagnostic distribution was as follows: 68 patients $(70.1 \%)$ consulted due to cough headache, $11(11.3 \%)$ due to exertional headache and $18(18.6 \%)$ due to sexual headache. A total of 28 patients $(41.2 \%)$ out of 68 were diagnosed of primary cough headache, while the remaining $40(58.8 \%)$ had secondary cough headache, always due to structural lesions in the posterior fossa, which in most cases was a Chiari type I malformation. In seven patients, cough headache was precipitated by treatment with angiotensin-converting enzyme inhibitors. As compared to the primary variety, secondary cough headache began earlier (average 40 vs. 60 years old), was located posteriorly, lasted longer (5 years vs. 11 months), was associated with posterior fossa symptoms/signs and did not respond to indomethacin. All those patients showed difficulties in the cerebrospinal fluid circulation in the foramen magnum region in the dynamic MRI study and preoperative plateau waves, which disappeared after posterior fossa reconstruction. The mean age at onset for primary headaches provoked by physical exercise and sexual activity began at the same age (40 years old), shared clinical characteristics (bilateral, pulsating) and responded to beta-blockers. Contrary to cough headache, secondary cases are rare and the most frequent etiology was subarachnoid bleeding. In conclusion, these conditions account for a low proportion of headache consultations. These data show the total separation between cough headache versus headache due to physical exercise and sexual activity, confirm that these two latter headaches are clinical variants of the same entity and illustrate the clinical differences between the primary and secondary provoked headaches. 
Keywords Chiari malformation - Cough headache . Exertional headache $\cdot$ Sexual headache

\section{Introduction}

Headaches provoked by cough, physical exercise (prolonged) and sexual activity can be primary or secondary to an intracranial structural lesion. These headaches have not been clearly defined until recent years. Already in 1932, Tinel was the first author who described several patients with intermittent, paroxysmal headaches brought on by exertion or manouvers capable of increase intrathoratic pressure [1]. In 1956, Sir Charles Symonds reported 27 patients with short-duration headaches provoked by Valsalva manouvers: cough, laughing, defecation or sudden postural changes [2]. He divided these patients into two groups: the first included 6 cases in which he was able to demonstrate an intracranial structural lesion, whereas the second comprised of the remaining 21 patients with no demonstrable lesion. Therefore, Symonds was the first author delimitating "benign" cough headache that is not related to any structural lesion. In spite of these findings, the first big Rooke's series of headache provoked by exertion was a mixture of headaches provoked by cough, prolonged exercise and sexual activity [3]. Rooke's influence remained until the 1990s. In 1991, Sands et al. [4] grouped together 219 cases of headache provoked by cough or by exertion. In this paper, Sands et al. found that one out of five cases was secondary to an intracranial lesion, usually located in the posterior fossa. After the initial descriptions, headache precipitated by sexual activity was well defined. Three classical types of headache related to sexual activity were described. Type 1 sexual headache is considered a variety of tension-type headache, while type 3 sexual headache is due to low cerebrospinal pressure, possibly due to a dural tear related to physical exercise. The true sexual headache, also called type 2 or vascular headache, appears suddenly during orgasm and is probably related to hemodynamic changes [5].

The relationship between these three provoked headaches is not totally clear. In Lance's experience, there was no relationship between headache provoked by prolonged physical exercise and (type 2) sexual headache [6]. By contrast, Silbert et al. [7] found that $40 \%$ of those patients with sexual headache also experienced headache with prolonged physical exercise. Things became clearer in 1996 when we published our retrospective experience with 72 patients who had consulted us due to these headaches [8]. Regardless of the different precipitants, we were able to separate the three headache subtypes clinically. Moreover, we found that the clinical characteristics of the benign headaches, for the three varieties, were different to those of symptomatic headaches, which could allow us to have a preliminary etiological diagnosis from clinical semeiology. In fact, the three headaches were clearly defined and separated in the current IHS classification [9]. An important limitating factor of all these works, including our series, was that data were collected restrospectively and that most patients were not studied with MRI techniques.

In this work, our aim was to have a better knowledge of these provoked headaches with a prospective clinical study an adequate follow-up and including the modern diagnostic techniques.

\section{Patients and methods}

Clinical evaluation

Between 1997 and 2006 (both years inclusive) all patients consulting due to headache precipitated by cough or other Valsalva manouvers, prolonged physical exercise and sexual activity were prospectively subjected to a structured interview. Patients were diagnosed after a detailed neurologic and medical history and clinical examination by physicians experienced in headache diagnosis (JP or AO). We also recorded in depth the clinical characteristics of headaches, including precipitants, localization, quality, duration, age at onset, associated symptoms, and effects of treatments. The patients were followed-up for a minimum of 1 year.

\section{Radiological studies}

A cranio-cervical MRI was obtained in all patients with cough headache. Chiari type I malformation was diagnosed when downward displacement of cerebellar tonsils below the foramen magnum was $>3 \mathrm{~mm}$. CSF dynamics were studied with MRI in Chiari type I patients. In patients with headache provoked by prolonged physical exercise or sexual activity, an immediate CT scan was always obtained. A lumbar tap was performed in selected cases. All these patients ultimately had an MRI and angioMRI study.

\section{Treatment protocol}

All symptomatic patients diagnosed as primary cough headache were treated with indomethacin. Those with primary headache due to prolonged physical exercise or sexual activity were treated initially with beta-blockers (propranolol or nadolol). Indomethacin was given if there were contraindications or no response to beta-blockers. 
All patients with secondary provoked headaches were evaluated by the Service of Neurosurgery (RM).

\section{Results}

A total of 6,412 patients consulted due to headache during the 10 years of the study. The number of patients who had consulted due to any of these headaches was 97 (1.5\% of all headaches). Diagnostic distribution was as follows: 68 patients $(70.1 \%)$ consulted due to cough headache, 11 (11.3\%) due to exertional headache and 18 (18.6\%) due to sexual headache (Fig. 1).

Cough headache

Those 68 patients diagnosed as having cough headache accounted for $1.1 \%$ of all the new consultations due to headache in the study period. A total of 28 patients $(41.2 \%)$ were diagnosed as primary cough headache, while the remaining $40(58.8 \%)$ were diagnosed as secondary cough headache (Fig. 1).

Primary cough headache

Among the 28 patients with primary cough headache, 18 $(64 \%)$ were women and $10(36 \%)$ men. The average age at onset was 60 years (range $22-80$ years). Predominant pain location was: hemicranial (50\% of cases), bilateral $(39 \%)$ and occipital-suboccipital (11\%) (Fig. 2). Pain quality was described as electrical ( $22 \%$ of cases), explosive $(17 \%)$, pressing (17\%) and as a mixture of all these (44\%) (Fig. 3 ). The duration of pain was seconds ( $78 \%$ of cases), more than $1 \mathrm{~min}(11 \%)$ and variable (sometimes seconds, sometimes $>1 \mathrm{~min}$ ) in the remaining $11 \%$. The mean duration of the symptomatic phase was 11 months (range 1-42 months). Regarding specific precipitants, in $100 \%$ of cases pain was provoked by cough, in $56 \%$ of cases by sudden postural movements, in $39 \%$ by weight lifting, in $33 \%$ by laughing and in $22 \%$ of cases by defecating. In the majority (24 patients, $86 \%$ ), there were no accompanying symptoms; the remaining 4 patients (14\%) complained of mild dizziness. Ten patients $(36 \%)$ suffered from arterial hypertension. Seven patients were receiving angiotensinconverting enzyme inhibitors (usually captopril), two calcium antagonists and the remaining one was not taking any antihypertensive. Regarding treatment, those seven patients treated with captopril and derivatives clearly improved when they stopped taking these drugs on retiring these drugs and ten did not require treatment due to spontaneous resolution or because the frequency of the attacks became low. The nine patients who were treated with indomethacin clearly responded to doses ranging from 50 to $100 \mathrm{mg}$ daily. Indomethacin was stopped every 2-3 months. This drug was required for a maximum period of 5 months.

\section{Secondary cough headache}

Among the 40 patients with secondary cough headache, 28 $(70 \%)$ were women and $12(30 \%)$ men. The mean age at onset was 44 (range 21-59). The predominant pain location was: occipital-suboccipital (72\% of cases), hemicranial (14\%) and bilateral (14\%) (Fig. 2). Pain quality was variable: pressing (31\% of cases), explosive $(21 \%)$, electrical (21\%) and a mixture of all of them (24\%) (Fig. 3). The
Fig. 1 Diagnostic distribution of patients who consulted due to headache. $H A$ headache, $S A H$ subarachnoid haemorrhage
6412 patients consult due to HA

$97(1.5 \%)$ consult due to provoked HA

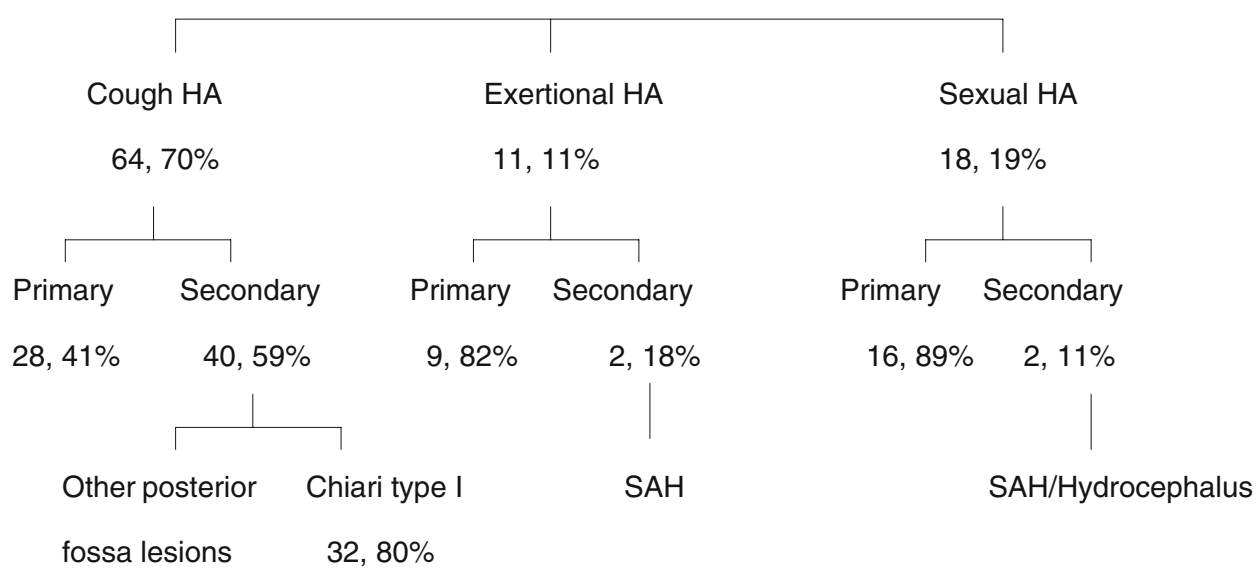




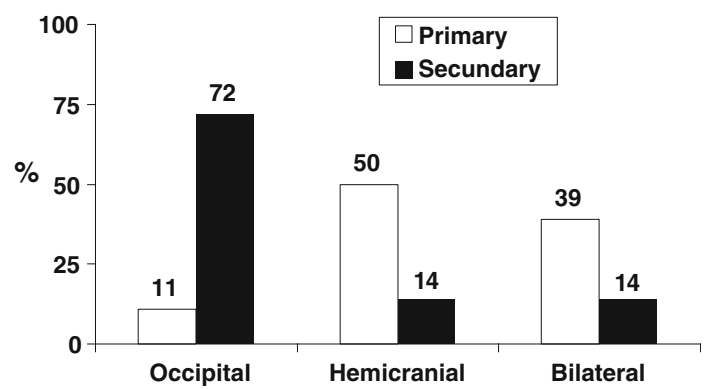

Fig. 2 Location of pain in primary and secondary cough headache. Most patients with secondary cough headache showed occipital pain location

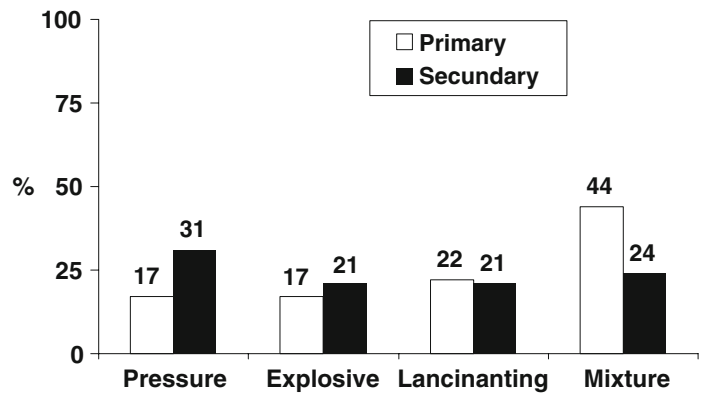

Fig. 3 Quality of pain in patients with primary versus secondary cough headache

duration of pain was as follows: seconds in $45 \%$ of cases, $>1 \mathrm{~min}$ in $10 \%$ and variable (sometimes seconds, sometimes $>1 \mathrm{~min}$ ) in the remaining $45 \%$. The average duration of the symptomatic phase was 5 years (maximum 30 years). Regarding pain precipitants, in $72 \%$ of cases pain was brought on by cough, in $41 \%$ by postural movements, in $31 \%$ by laughing and in $17 \%$ by defecation. Only seven patients (17.5\%) did not show any other accompanying symptoms or signs. The remaining 33 patients $(82.5 \%)$ showed posterior fossa semeiology: dizziness (13 patients, 33\%), unsteadiness $(9,23 \%)$, facial and upper limbs numbness $(18 \%)$, vertigo $(3,8 \%)$, syncope $(2,5 \%)$ and other symptoms $(10,25 \%)$. Five patients received indomethacin, in all cases without response.

Regarding etiologies, 32 patients (80\% of cases) had a Chiari type I malformation (Fig. 4). The remaining 8 patients had other posterior fossa structural lesions: 3 arachnoid cysts, 2 dermoid tumours, 2 meningiomas and one os odontoideum (Fig. 5). Pain disappeared in the 9 patients whose Chiari type I malformation was operated on with successful anatomical reconstruction of the posterior fossa (Fig. 4). All those patients showed a cerebrospinal fluid stop (anterior, posterior or both) in the foramen magnum region in the dynamic MRI study (Fig. 6). In addition, the intracranial preoperatory pressure registry disclosed clear plateu waves (Fig. 7), which disappeared after posterior fossa reconstruction.

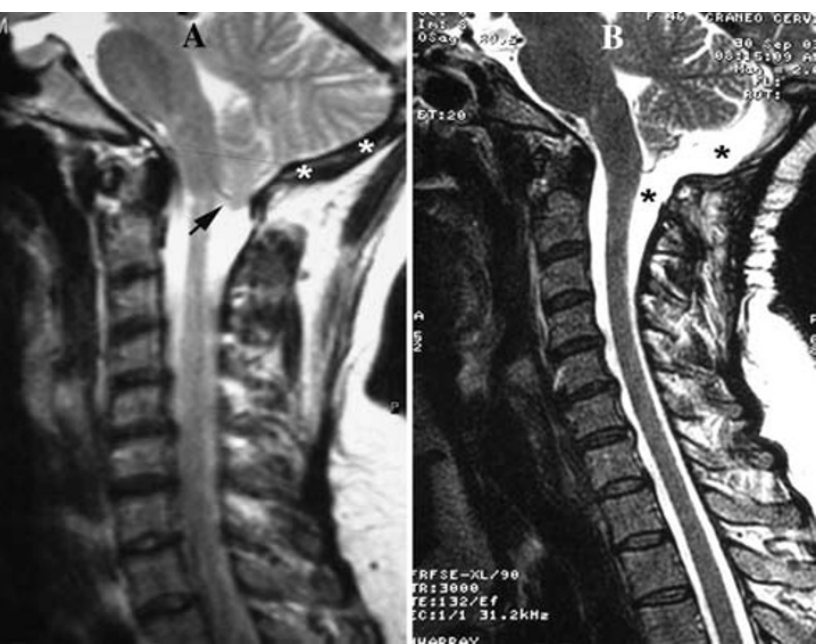

Fig. 4 Preoperative (a) and postoperative (b) T2-weighted sagittal MRI of a 36-year-old woman with cough headache. Note the presence of tonsilar descent (arrow) and flattening of posterior fossa (asterisks) and the absence of cisterna magna. b After posterior fossa reconstruction, notice the appearance of cisterna magna with restitution of cerebrospinal fluid transit (asterisks) with upward migration of the tonsils

Headache precipitated by prolonged physical exercise

In the study period, 11 patients consulted due to headache provoked by prolonged physical exercise. Nine $(82 \%)$ were diagnosed as primary and two (18\%) as secondary exertional headache. These two cases were symptomatic to subarachnoid bleeding in two men aged 16 and 67-yearold.

The mean age at onset for primary exertional headache was 40 (range 17-53). Eight (89\% of patients) were males. Pain location was bilateral in 8 cases and hemicranial in one. In all cases, pain was or had a pulsating component. Pain duration ranged between $1 \mathrm{~h}$ and 4 days (median without treatment $4 \mathrm{~h}$ ). The duration of the symptomatic period ranged between 1 day and 4 years (median 2 months). In all cases the precipitant was prolonged physical exercise; three patients also admitted sexual headache. Regarding concomitant disorders, 3 (33\%) of the nine patients met criteria for migraine without aura and one arterial hypertension. Five of these nine patients required pharmacological treatment. All responded to beta-blockers (nadolol or propranolol) at $1-2 \mathrm{mg} / \mathrm{kg}$ per day. The period of treatment ranged from a minimum of two and a maximum of 6 months.

\section{Sexual headache}

Eighteen patients consulted due to headache provoked by sexual activity. There were only two patients with secondary sexual activity: a male aged 27-year-old with 
Fig. 5 Examples of patients consulting due to secondary cough headache who were not diagnosed of Chiari type I malformation. Notice the identical location of the three tumours

\section{Posterior fossa occupying lesions}
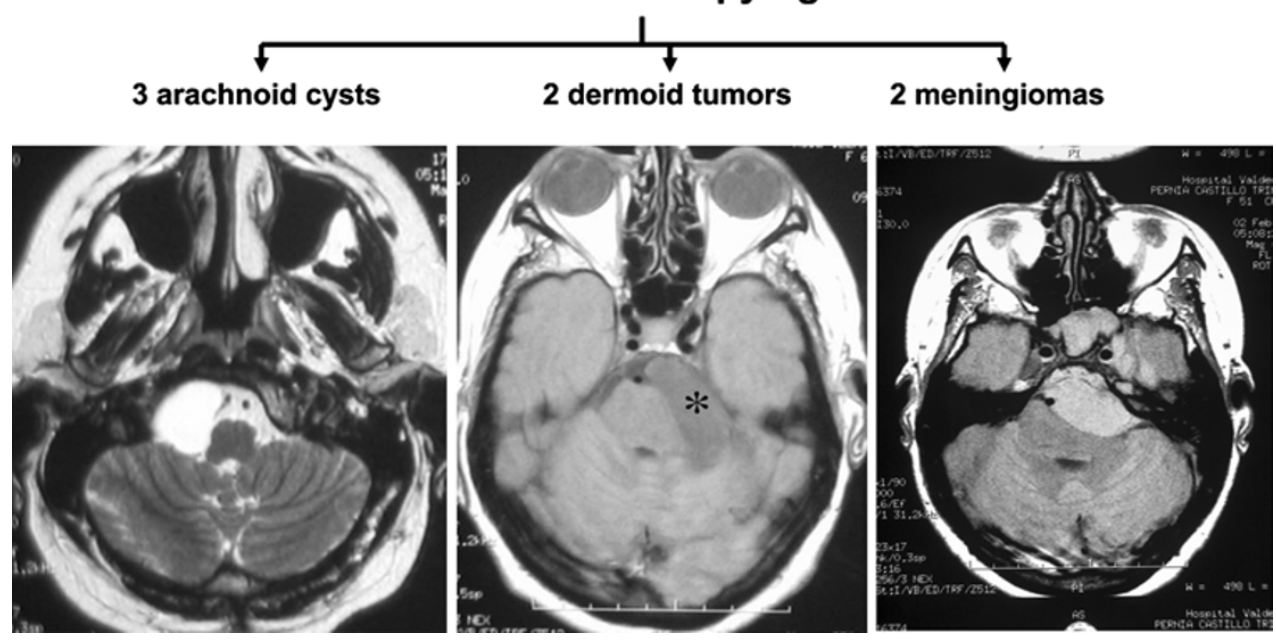
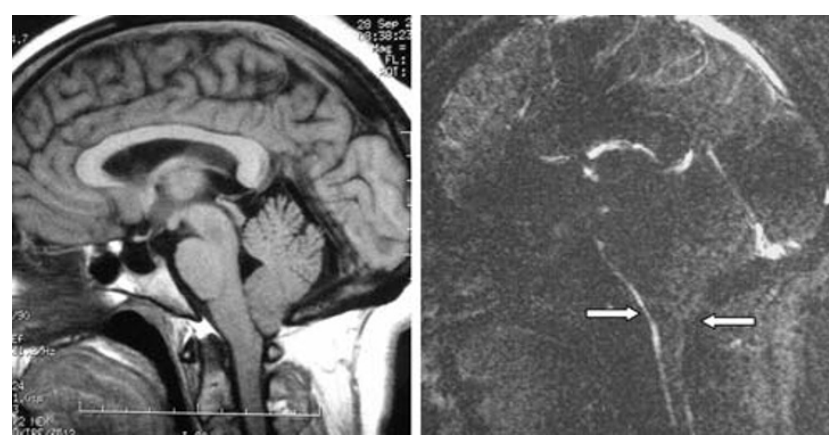

Fig. 6 Cine-phase MRI of a 33-year-old female with Chiari type I presenting as cough headache showing difficulties in cerebrospinal fluid circulation both anteriorly and posteriorly in the foramen magnum region (arrows)

hydrocephalus due to Silvian aqueductal stenosis and a female aged 20-year-old with a cervical arteriovenous malformation (Fig. 8).

The mean age at onset of the 16 patients with primary exertional headache was 40 (range 16-58). Thirteen ( $81 \%$ of patients) were males. Pain location was bilateral ("over the temples") in 12 cases (75\%) and hemicranial in the remaining 4 (25\%). In all cases, pain was or had a pulsating component. Pain duration ranged between $1 \mathrm{~min}$ and 4 days (median $10 \mathrm{~min}$ ). Five patients referred to the following accompanying symptoms: nausea (3 cases), phono/ photophobia ( 2 cases) and eye tearing ( 1 case). The duration of the symptomatic period ranged between 20 days and 6 years (median 2 months). Regarding concomitant disorders, 5 (31\%) of the 9 patients met criteria for migraine without aura and $3(19 \%)$ had arterial hypertension. Five of these nine patients required pharmacological treatment. Nine patients were treated with beta-blockers (nadolol or propranolol) at $1-2 \mathrm{mg} / \mathrm{kg}$ per day. There was a clear response in 8 . Due to asthma and no response to betablockers, two patients were treated with indomethacin $(25 \mathrm{mg} / 8 \mathrm{~h})$ with excellent response. The period of treatment ranged from a minimum of 2 and a maximum of 6 months.

\section{Discussion}

Apart from the old study from the Mayo Clinic [3], this work contains the highest number of patients with exertional headaches in the literature. This is the only series
Fig. 7 Preoperatory intracranial pressure registry of a patient with cough headache and Chiari type I malformation in which abnormal waves of intracranial hypertension are seen. These high pressure waves

disappeared after successful surgery
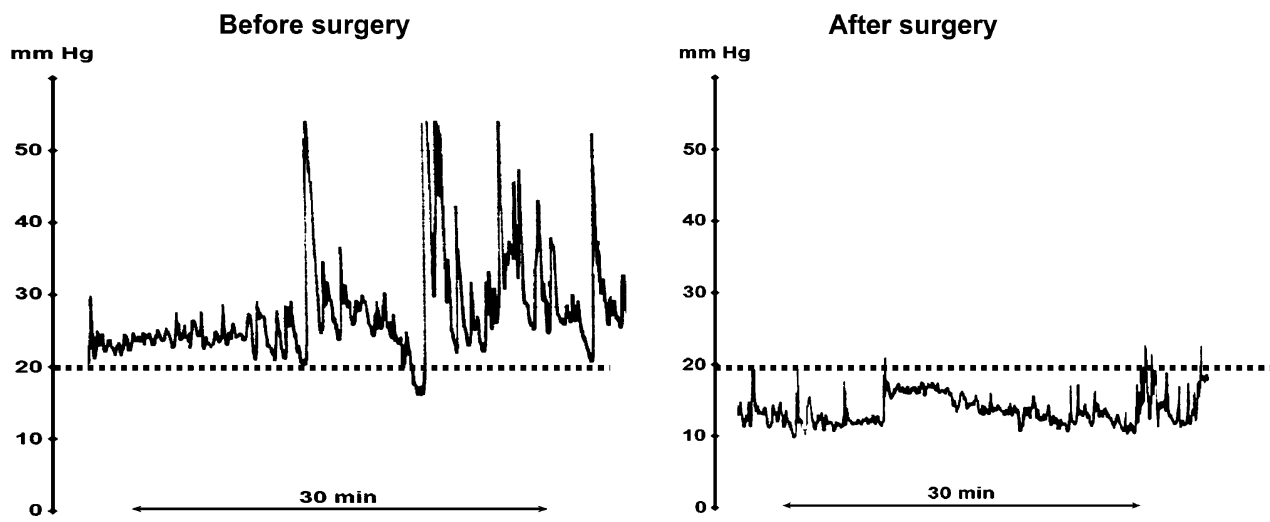

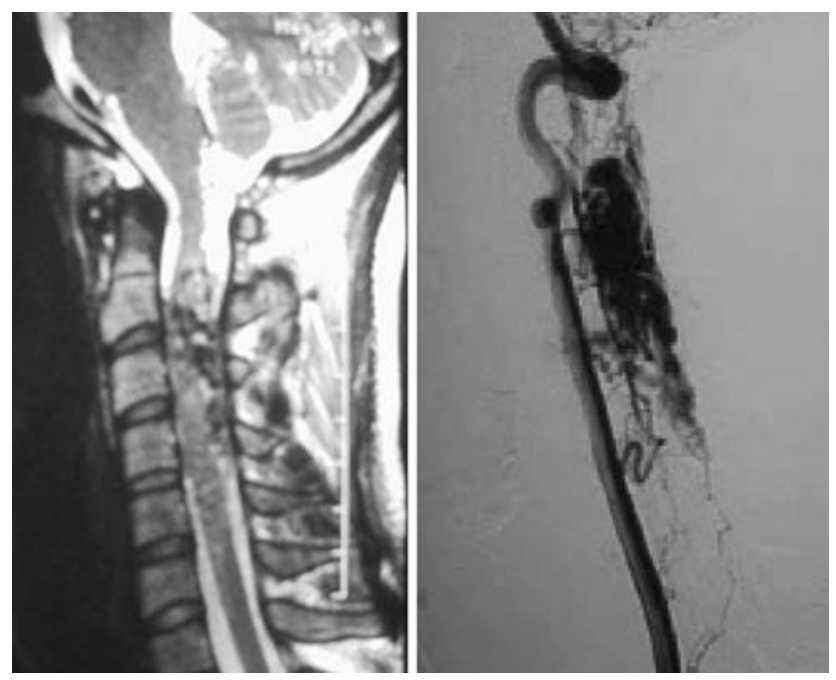

Fig. 8 MRI and angiography of a patient who consulted due to several episodes of sexual headache showing a cervical vascular arteriovenous malformation

with prospective and long-term follow-up and including modern radiological techniques. Our data confirm the total separation of cough headache from both headache due to prolonged physical exertion and sexual headache, as well as the clear clinical differences, for each provoked headache, between primary and secondary varieties.

The epidemiology of provoked headaches is unknown. Available data have shown that the prevalence of cough headache and headache related to prolonged physical exercise ranges from 0 to $2 \%$ in the general population $[10,11]$. Therefore, those are infrequent headaches when compared to migraine, with prevalence close to $20 \%$ in the general population. This is not an epidemiological survey. This series, however, offers an indirect idea of the frequency of these provoked headaches. Our data confirm that these headaches are infrequent, accounting for $1.5 \%$ of consultations due to headache in a Department of Neurology. Considering that $25 \%$ of all outpatient neurological consultations are due to headache, an easy calculation indicates that one out of 300-350 patients attending an outpatient general neurological clinic suffers from provoked headaches.

At least in terms of consultation, cough headache is the most frequent, accounting for $70 \%$ of provoked headaches. Sixty percent of cough headache cases were symptomatic to posterior fossa lesions. These numbers are clearly different to those coming from all previous series. Just $10 \%$ of Rooke's patients and 22\% of Symonds patients had intracranial structural lesions [2, 3]. In our retrospective series in which not all patients were studied with MRI, the proportion of symptomatic cases was lower [9]. These data show the relevance of such a prospective survey, with a high number of patients and including an MRI study, for an accurate classification of these patients and for a right differential diagnosis.

Primary cough headache is located in the occipital area in only one out of 10 cases, does not have a well-defined quality, usually lasts seconds and is always precipitated by cough. In contrast to the primary variety, symptomatic cough headache shows an occipital location in 7 out of 10 cases, usually lasts longer (more than $50 \%$ of cases last at least $1 \mathrm{~min}$ ) and is not precipitated by cough (but by other Valsalva manouvers) in almost one-third of cases. Even though they share the same precipitant, primary and secondary cough headache are clinically different. Secondary cough headache begins at an average of 16 years earlier than primary cough headache (mean 44 vs. 60 years old). Because of this, primary cough headache should be suspected in old patients and viceversa, secondary cough headache is the first possibility in young patients. Interestingly, our data do not confirm the male predominance found in previous works, including our series [9], in primary cough headache. Gender, therefore, is not a clue for etiological diagnosis, as it was previously proposed. There are several potential explanations for this discrepancy. First, we should bear in mind that this is the most accurate series in terms of clinical and radiological methodology. Second, and probably due to recent changes in life style, the gender distribution of headaches, such as cluster headache, with a clear male predominance is equilibrating. Five out of 7 patients taking angiotensin-converting enzyme inhibitors due to arterial hypertension were females, which contributed to equal gender distribution.

There are other clinical clues useful in the differential diagnosis between primary and secondary cough headache. Patients with secondary cough headache refer to a long headache history (average 5 years) as compared to primary cough headache cases (average 11 months). In addition, while the vast majority of cases with primary cough headache did not show other accompanying symptom/ signs, more than $80 \%$ of cases diagnosed as secondary cough headache had posterior fossa semeiology. We cannot be very strict in this point, however, as 6 of our patients with Chiari type I malformation only had cough headache without other subjective complaints orabnormal findings on examination. Finally, all treated primary cough headache cases responded to indomethacin, while this drug was of no value in secondary cases in our experience $[12,13]$.

Regarding the etiology of secondary cases, without exception they all had posterior fossa lesions. In $80 \%$ of cases, this lesion was a Chiari type I malformation. In the remaining cases, other posterior fossa occupying lesions, usually benign tumors, were found in the foramen magnum area. These findings explain the low incidence of structural lesions in classical series, as Chiari type I malformation can only be diagnosed with certainty by means of a 
cranio-cervical MRI study. This series also contributes to clarify further the pathophysiology of secondary cough headache. In all patients with secondary cough headache there was a restricted circulation of cerebrospinal fluid in the foramen magnum area, anterior, posterior or at both levels. Those patients requiring decompressive surgery showed high cerebrospinal pressure waves, which disappeared, together with the cough headache, after reconstructive surgery. These facts indicate that headache is secondary to an abnormal tonsilar descent, which increase with Valsalva maneouvers, because of: (1) compression/irritation of the upper cervical roots (very pain sensitive), and (2) a transient phenomenon of intracranial hypertension [14-18]. The pathophysiology of primary cough headache is not known. The possibility of a sudden increase in venous pressure being sufficient itself to cause headache due to an increase in brain volumen has been proposed [19]. There should be other contributing factors, however, such as a hypersensitivity of some receptors, sensitive to pressure, hypothetically localized on the venous vessels [20]. One of the potential etiologies for this transient receptor sensitization could be a hidden or previous infection [21]. A new and interesting finding of this series is the fact that in some patients cough headache coincided in time with the intake of angiotensin-converting enzyme inhibitors, drugs with a demonstrated coughinducing effect [22]. Finally, Chen and co-workers have recently found that patients with primary cough headache are associated with a more crowded posterior cranial fossa, which may be a further contributing factor for the pathogenesis of this headache syndrome [23].

This series also shows that cough headache is different to headache caused by prolonged physical exercise and sexual headache. Leaving the precipitant aside, differences are multiple. The age at onset for primary cough headache (60 years old) was 20 years higher than that of the other two exertional headaches. Male predominance was striking (4/1) for exertional and sexual headaches, but did not occur for primary cough headache, which concurs with previous observations [9, 24]. The pain characteristics (bilateral and pulsating), comorbidity (migraine without aura), response to preventive treatment (beta-blockers) and, above all, the fact that some patients admitted to a history of both sexual and exertional headache confirm Silbert et al. experience, that these two headaches share many aspects [7]. These data are not surprising for us. Sexual intercourse can be considered as a prolonged physical exercise and that is the reason why some patients with sexual headache do not experience exertional headache is that they do not practice any other exercise. The pathophysiology of these two primary headaches is unknown, though sudden hemodynamic changes have been proposed as an explanation [25]. The etiologies for secondary cases of these two headaches were subarachnoid bleeding and, in one case, hydrocephalus. Therefore, diagnostic investigation in these headaches must begin first with a neuroimaging study (CT or MRI) to rule out subarachnoid bleeding followed by an angioMRI. Conventional angiography and/or lumbar tap would be indicated only in those punctual cases with a high suspicion of bleeding despite a negative angioMRI, but not as a routine.

\section{References}

1. Tinel J (1932) La céphalée a l'effort. Syndrome de distension douloureuse des veines intracraniennes. Médecine (Paris) 13:113-118

2. Symonds C (1956) Cough headache. Brain 79:557-568

3. Rooke ED (1968) Benign exertional headache. Med Clin North Am 52:801-808

4. Sands GH, Newman L, Lipton R (1991) Cough, exertional and other miscellaneous headaches. Med Clin North Am 75:733-747

5. Headache Classification Committee of the International Headache Society (1988) Classification and diagnostic criteria for headache disorders, cranial neuralgias and facial pain. Cephalalgia 8(Suppl 7):19-28

6. Lance JW (1991) Solved and unsolved headache problems. Headache 31:439-445

7. Silbert PL, Edis RH, Stewart-Wynne EG et al (1991) Benign vascular sexual headache and exertional headache: interrelationships and long term prognosis. J Neurol Neurosurg Psychiatry 54:417-421

8. Pascual J, Iglesias F, Oterino A, Vázquez-Barquero A, Berciano J (1996) Cough, exertional, and sexual headaches. An analysis of 72 benign and symptomatic cases. Neurology 46:1520-1524

9. Headache Classification Committee of the International Headache Society (2004) The international classification of headache disorders, 2nd edn. Cephalalgia 24(Suppl 1):1-160

10. Rasmussen BK, Olesen J (1992) Symptomatic and nonsymptomatic headaches in a general population. Neurology 42:12251231

11. Sjaastad O, Pettersen H, Bakketeig LS (2001) The Vaga study; epidemiology of headache I: the presence of ultrashort paroxysms. Cephalalgia 21:207-215

12. Mathew NT (1981) Indomethacin responsive headache syndromes. Headache 21:147-150

13. Diamond S (1982) Prolonged benign exertional headache: its clinical characteristics and response to indomethacin. Headache 22:96-98

14. Williams B (1976) Cerebrospinal fluid changes in response to coughing. Brain 99:331-346

15. Williams B (1980) Cough headache due to craniocerebrospinal pressure dissociation. Arch Neurol 37:226-230

16. Sansur CA, Heiss JD, DeVroom LH, Eskioglu E, Ennis R, Oldfield EH (2003) Pathophysiology of headache associated with cough in patients with Chiari malformation. J Neurosurg 98:453458

17. Pujol A, Roig C, Capdevila A et al (1995) Motion of the cerebellar tonsils in Chiari type I malformation studied by cine-phase contrast MRI. Neurology 45:1746-1753

18. Pascual J, Oterino A, Berciano J (1992) Headache in type I Chiari malformation. Neurology 42:1519-1521

19. Wang SJ, Fuh JL, Lu SR (2000) Benign cough headache is responsive to acetazolamide. Neurology 55:149-150

20. Raskin NH (1997) Short-lived head pains. Neurol Clin 15:143-152 
21. Wolff HG (1963) Pain-sensitive structures within the cranial cavity. In: Headache and other head pain, 2nd edn. New York, Oxford University Press, pp 53-95

22. Parish RC, Miller LJ (1992) Adverse effects of angiotensin converting enzyme inhibitors. An update Drug Saf 7:14-31

23. Chen YY, Lirng JF, Fuh JL, Chang FC, Cheng HC, Wang SJ (2004) Primary cough headache is associated with posterior fossa crowdedness: a morphometric MRI study. Cephalalgia 24:694-699
24. Frese A, Eikermann A, Frese K, Schwaag S, Husstedt IW, Evers S (2003) Headache associated with sexual activity: demographics, clinical features, and comorbidity. Neurology 23:796-800

25. Evers S, Schmidt O, Frese A, Husstedt IW, Ringelstein EB (2003) The cerebral hemodynamics of headache associated with sexual activity. Pain 102:73-78 